\title{
SET OF UNIQUENESS ON NONCOMMUTATIVE LOCALLY COMPACT GROUPS
}

\author{
MAREK BOZ̊EJKO
}

\begin{abstract}
Using the terminology of P. Eymard we adapt the notion of set of uniqueness to noncommutative case and we show that every compact and residual set in a locally compact nondiscrete group is a set of uniqueness.
\end{abstract}

In this note we shall prove that every compact and residual (i.e. including no nonempty perfect set) set in locally compact nondiscrete group $G$ is a set of uniqueness. In the particular case, every countable compact set is a set of uniqueness. For the case when the group $G$ is a torus, this is a classical result of W. H. Young (see [1], [3], [8]). For commutative groups that fact was proved by L. H. Loomis [4].

We refer to $P$. Eymard [2] for the basic definitions, properties and theorems of $A(G), B(G), V N(G)$ and $C_{\rho}^{*}(G)$.

Now we recall some facts. $C_{\rho}^{*}(G)$ and $V N(G)$ are, respectively, the $C^{*}$ algebra and the von Neumann algebra generated by the operators $\rho(f)$ on $L^{2}(G)$, where $\rho$ is the left regular representation of the group $G$ and $f$ is an arbitrary function on $G$ with the compact support.

As in [2], we denote $C^{*}(G)$, the enveloping $C^{*}$-algebra of $L^{1}(G)$, i.e. the completion of the algebra $L^{1}(G)$ with respect to the norm

$$
\|f\|_{C^{*}(G)}=\sup \{\|\pi(f)\|: \pi \in \Sigma\}
$$

where $\Sigma$ denotes the space of all ${ }^{*}$-representations of $L^{1}(G)$ on a Hilbert space.

The Fourier-Stieltjes algebra $B(G)$ consists of all finite complex linear combinations of continuous positive definite functions on $G$. As shown in [2], $B(G)$, as the dual of $C^{*}(G)$, is a commutative Banach algebra with unit (the multiplication is defined pointwise). The algebra $A(G)$ is defined as the norm closure in $B(G)$ of the functions from $B(G)$ with compact supports. We note also that $V N(G)$ is the dual of $A(G)$.

We recall also from [2] that if $T \in V N(G)$ and $u \in B(G)$, we can define the multiplication $u \cdot T \in V N(G)$ in the following way: for every function $v$ from $A(G), u \cdot T$ is a functional on $A(G)$ such that $\langle v, u \cdot T\rangle=\langle u v, T\rangle$.

Received by the editors January 14, 1976.

AMS (MOS) subject classifications (1970). Primary 43A55, 22D25.

$K e y$ words and phrases. Set of uniqueness, $C_{\rho}^{*}(G)$-algebra. 
As in [2], the element $a$ from $G$ belongs to the support of the operator $T \in V N(G)(a \in \operatorname{supp}(T))$, if the following equivalent statements hold:

(i) For every neighborhood $V$ of $a$, there exists a function $u \in A(G)$ with support in $V$ such that $\langle u, T\rangle \neq 0$.

(ii) If $u \in A(G)$ and $u \cdot T=0$, then $u(a)=0$.

Now we can introduce in general case the following

Definition 1. Let $E$ be a compact subset of $G$; a set $E$ is called $a$ set of uniqueness if $T \in C_{\rho}^{*}(G)$ and $\operatorname{supp}(T) \subseteq E$ imply $T=0$.

We note that if the group $G$ is commutative and $\hat{G}$ is the dual group, then $C_{\rho}^{*}(G)$ and $V N(G)$, respectively, coincide with the spaces $C_{0}(\hat{G})$ and $L^{\infty}(\hat{G})$, and the support of $T \in V N(G)$ coincides with the spectrum of its inverse Fourier transform which is an element of $L^{\infty}(\hat{G})$.

Thus, for the commutative groups, Definition 1 reduces to the usual notion of a set of uniqueness (see e.g. Y. Meyer [5]).

Proposition 1. If the group $G$ is nondiscrete, then $C_{\rho}^{*}(G) \cap C_{\rho}^{*}\left(G_{d}\right)=\{0\}$, where $G_{d}$ is the group $G$ with discrete topology.

Proof. We recall that every $S \in C_{\rho}^{*}\left(G_{d}\right)$ is of the form $S=\rho(F), F=$ $S\left(\delta_{e}\right),\|S\|_{\rho} \geqslant\|F\|_{2}={ }^{\mathrm{df}}\|S\|_{2}$, and $S=0$ if and only if $\|S\|_{2}=0$. Now we show that

$$
\operatorname{dist}\left(S, C_{\rho}^{*}(G)\right) \geqslant\|S\|_{2} \quad \text { for } \quad S \in C_{\rho}^{*}\left(G_{d}\right) .
$$

Using the density argument it suffices to prove that

$$
\|T-\rho(f)\|_{\rho} \geqslant\|T\|_{2}
$$

for every continuous function $f$ on $G$ with compact support $T$ of the form $T=\sum_{n=1}^{N} a_{n} \rho\left(x_{n}\right)$. Let $\left\{V_{\alpha}\right\}$ be a family of symmetric neighborhoods of identity such that the Haar measure of $V_{\alpha}$ tends to zero, and put $h_{\alpha}=$ $|V|^{-1 / 2} \chi_{v_{\alpha}}$, where $\chi_{v_{\alpha}}$ is the characteristic function of the set $V_{\alpha}$. Since the net $u_{\alpha}=\left|V_{\alpha}\right|^{-1 / 2} h_{\alpha}$ is an approximate unit in $L^{2}(G)$, we obtain

$$
\lim _{\left|V_{\alpha}\right| \rightarrow 0}\left\|f * h_{\alpha}\right\|_{2}=0
$$

Note also that

$$
\lim _{\left|V_{\alpha}\right| \rightarrow 0}\left\|T\left(h_{\alpha}\right)\right\|_{2}=\|T\|_{2}
$$

and that fact gives (2).

COROllary 1. The algebra $C_{\rho}^{*}(G)$ has a unit if and only if the group $G$ is discrete.

Now we introduce the following auxiliary definition (see also [4]).

Definition 2. Let $T \in V N(G)$ and $x \in G$. We say that $T$ belongs locally to $C_{\rho}^{*}\left(G_{d}\right)$ at the point $x\left(T \in C_{\rho}^{*}(x)\right)$ if there exists a function $u \in A(G)$ such that $U(x) \neq 0$ and $u \cdot T \in C_{\rho}^{*}\left(G_{d}\right)$. 
RemarK. Since every function from $A(G)$ is continuous, so for every $T$ from $V N(G)$ the set $A_{T}=\left\{x \in G: T \in C_{\rho}^{*}(x)\right\}$ is open.

Lemma 1. Let $T \in V N(G)$ and the support of $T$ be compact. Then:

(a) if $u_{1}$ and $u_{2}$ from $B(G)$ are identical on a neighborhood of the support of $T$, then $u_{1} T=u_{2} T$.

(b) $T \in C_{\rho}^{*}\left(G_{d}\right)$ if and only if, for every $x \in G$, we have $T \in C_{\rho}^{*}(x)$.

The proof is the same as in Abelian case (see [2], [4]).

Lemma 2. $C_{\rho}^{*}(G)$ and $C_{\rho}^{*}\left(G_{d}\right)$ are $B(G)$-modules.

The proof follows from the following two facts (see [2]):

(1) If $T=\rho(\mu)$, where $\mu$ is a Borel regular measure on $G$ and $v \in B(G)$, then $v \cdot \rho(\mu)=\rho \cdot(v \mu)$.

(2) If $T \in V N(G)$ and $u \in B(G)$, then $\|u \cdot T\|_{\rho}<\|u\|_{B} \cdot\|T\|_{\rho}$.

LEMMA 3. If the support of $T \in V N(G)$ is compact, then $T \in C_{\rho}^{*}(x)$ for every $x$ which is not in the support of $T$.

Proof. From the regularity of the algebra $A(G)$ there exists a function $u \in A(G)$ such that $u=0$ on a neighborhood of the support of $T$ and $u(x) \neq 0$. So by the Lemma $1(\mathrm{a}), u \cdot T=0$; but this means that $T \in C_{\rho}^{*}(x)$.

We now can prove the following

Proposition 2. Let $T \in V N(G)$ and let $T$ have a compact support. Then if $T \in C_{\rho}^{*}(x)$ for every $x \neq e$, then $u \cdot T \in C_{\rho}^{*}\left(G_{d}\right)$ for every $u \in B(G)$ such that $u(e)=0$.

Proof. Since the support of $T$ is compact, there exists a function $v \in A(G)$ such that $v=1$ on the neighborhood of the support of $T$. Hence by Lemma 1(a), $v \cdot T=T$. But the Fourier algebra $A(G)$ is an ideal in $B(G)$ so $u v$ is in $A(G)$ and $u v(e)=0$. Now we can approximate $u v$ in $A(G)$ norm by a function $g \in A(G)$ which vanishes in a neighborhood of the identity. (See [2, Corollary (4.11)].) Because $\operatorname{supp}(g \cdot T) \subset \operatorname{supp}(g) \cap \operatorname{supp}(T)$, so the identity is not in the support of $g \cdot T$; hence, by Lemma $3, g \cdot T \in C_{\rho}^{*}(e)$. On the other hand, it is obvious that $g \cdot T \in C_{\rho}^{*}(x)$ for every $x \neq e$, so by Lemma 1(b), $g \cdot T \in C_{\rho}^{*}\left(G_{d}\right)$. Since $u \cdot T=(u v) \cdot T$ and $C_{\rho}^{*}\left(G_{d}\right)$ is complete in the operator norm, Proposition 2 follows.

COROLlaRY 2. Let $G$ be a locally compact nondiscrete group. If

$$
T \in C_{\rho}^{*}(G) \cap C_{\rho}^{*}(x) \text { for every } x \neq e,
$$

and $T$ has a compact support, then $T=0$.

Proof. From Proposition 2 we have that $u \cdot T \in C_{\rho}^{*}\left(G_{d}\right)$ for every $u \in$ $B(G)$ such that $u(e)=0$. But also from Lemma $2, u \cdot T \in C_{\rho}^{*}(G)$, so by Proposition 1 we have $u \cdot T=0$. From condition (ii) of the definition of the 
support of the operator $T$, we obtain $u(a)=0$ for every $u \in A(G)$ such that $u(e)=0$ and every point $a \in \operatorname{supp}(T)$. But if $T \neq 0$, then we can consider two cases: either $\operatorname{supp}(T)=\{e\}$ or $\operatorname{supp}(T) \supset\{e, a\}$ for some $a \neq e$. In the first case $T=\lambda I$ (see [2, Theorem 4.9]). But $T \in C_{\rho}^{*}(G)$, so by Proposition 1 we get a contradiction; the second case is also impossible because we can take a function $u \in A(G)$ such that $u(a) \neq 0$ and $u(e)=0$.

Now we are in position to prove

THEOREM. If $E$ is compact and residual in a nondiscrete group $G$, then $E$ is a set of uniqueness.

Proof. Let $T$ be in $C_{\rho}^{*}(G)$ with support in $E$. We know that the set $A_{T}$ is open and $A_{T}^{c}$, the complement of $A_{T}$ in $G$, is contained in the support of $T$. We shall prove that $A_{T}^{c}$ is empty.

Let $A_{T}^{c}$ be nonempty and let $x_{0}$ be an isolated point of $A_{T}^{c}$; we also can assume that $x_{0}=e$. From the regularity of $A(G)$, there exists a function $u \in A(G)$ such that $u(e) \neq 0$ and $u(x)=0$ for $x$ from $A_{T}^{c} \backslash\{e\}$. Hence by Lemma $3, u \cdot T \in C_{\rho}^{*}(x)$ for every $x \neq e$ and also $u \cdot T \in C_{\rho}^{*}(G)$. So by the Corollary $2 u \cdot T=0$, i.e. $e \notin A_{T}^{c}$ a contradiction. Hence $A_{T}^{c}=\varnothing$ and, by Lemma 1(b), $T \in C_{\rho}^{*}\left(G_{d}\right)$. Finally, using Proposition 1, we obtain $T=0$.

COROLlaRY 3. Every countable compact set in nondiscrete group is a set of uniqueness.

REMARK. Using the same argument as in Abelian case one can show that a finite union of sets of uniqueness is also a set of uniqueness.

\section{REFERENCES}

1. N. K. Bari, Trigonometric series, Vols. I, II, Fizmatgiz, Moscow, 1961; English transl., Macmillan, New York, 1964. MR 23 \# A3411; 30 \# 1347.

2. P. Eymard, L'algèbre de Fourier d'un groupe localement compact, Bull. Soc. Math. France 92 (1964), 181-236. MR 37 \#4208.

3. J.-P. Kahane et R. Salem, Ensembles parfaits et séries trigonométriques, Hermann, Paris, 1963. MR 28 \# 3279.

4. L. H. Loomis, The spectral characterization of a class of almost periodic functions, Ann. of Math. (2) 72 (1960), 362-368. MR 22 \#11255.

5. Y. Meyer, Algebraic numbers and harmonic analysis, North-Holland, Amsterdam, 1972.

6. W. Rudin, Fourier analysis on groups, Interscience, New York, 1962. MR 27 \#2808.

7. N. Varopoulos, Sets of multiplicity in locally compact abelian groups, Ann. Inst. Fourier (Grenoble) 16 (1966), fasc. 2, 123-158. MR 35 \#3379.

8. A. Zygmund, Trigonometric series, Monografie Mat., vol. 5, PWN, Warsaw, 1935; 2nd rev. ed. (reprinted with corrections and additions), Vols. I, II, Cambridge Univ. Press, New York, 1968. MR 38 \# 4882.

Institute of Mathematics, The Polish Academy of Sciences, Warsaw, Poland

Department of Mathematics, University of Wrockaw, Wrockaw, Poland 